\title{
Rural Development in a Function of Local Economic Development: Case Study of Municipality of Krushevo
}

\author{
Nikolche Jankulovski ${ }^{1}$, Emiliana Silva $^{2}$, Katerina Bojkovska $^{1} \&$ Angjelka Jankulovska ${ }^{1}$ \\ ${ }^{1}$ University "St. Kliment Ohridski”, Bitola, Republic of Macedonia \\ ${ }^{2}$ University of the Azores, Portugal \\ Correspondence: Nikolche Jankulovski, University "St. Kliment Ohridski”, Bitola, Republic of Macedonia. \\ E-mail: nikolcejankulovski@yahoo.com
}

Received: November 8, 2016

Accepted: November 17, 2016

Online Published: December 18, 2016

doi:10.5539/ijef.v9n1p162

URL: http://dx.doi.org/10.5539/ijef.v9n1p162

\begin{abstract}
The local economic development is a process in which the local authorities and partners from the business sector and the non-governmental sector work together to improve the business climate. Through positive financial results conditions for economic growth are created and the employment opportunities are increased. Most of the municipalities in the Republic of Macedonia are still at an early stage of development of the idea of local economic development, due to fact that the preparation of the municipal strategies and action plans are the only activities carried out in this direction so far.

The National Strategy for Agriculture and Rural Development for the period 2009-2013 stresses tourism and agriculture as priority areas for development of the country and analyzes the situation and the range of responsibilities of the Ministry of Agriculture, Forestry and Water Management. Parallel, the national programs facilitating agriculture and rural development in Macedonia in favor of global trends for creation of sustainability in rural regions through the development of agriculture and additional activities for families living or returning to rural areas (villages). The rural development through the few authentic examples in Macedonia already shows the first interest although the contours of the branch are not defined yet.

The rural development is able to a greater extent to meet the needs of the modern man that the urban environment is not able to satisfy. To clarify what can be these unmet needs and to answer the question of interest due to demand for this type of tourism, it is necessary to analyze contained rural tourism.
\end{abstract}

Keywords: absorption, administration, capacity, development

\section{Results with Discussion}

The need to determine the development and prospect of the rural economic development of the municipality of Krushevo was inspired from the increasing development of rural areas in Europe and worldwide. The rural areas in Europe and in the world revive and develop through the rural development with greater attendance by visitors or tourists. In terms of that, the objective of this master's thesis is to give a clearer picture of the status of the rural development in the Republic of Macedonia with special reference to the municipality of Krushevo, as well as with emphasis of the opportunities and prospects for improvement and modernization in future.

In order to ensure a more systematic and scientific foundation of the paper, several methods of work commonly used in economic analysis, resulting both in theoretical and in practical knowledge, are applied in its preparation. In the research of this master's thesis questionnaires for the respondents from the municipality of Krushevo were used. Questions about the current state of the rural development and tourism in the municipality, as well as questions about the possible perspectives, and the directions in which itcan be developed and promoted were asked in the questionnaires.

The rural development in the municipality Krushevo must be consistent with the objectives of national agricultural policy of the Republic of Macedonia, and they are focused on:

$\checkmark$ Provision of stable production of quality, cheap food and providing the population with sufficient quantities of food;

$\checkmark$ Increase of the competitiveness of agriculture; 
$\checkmark$ Provision of stable income for farmers;

$\checkmark$ Sustainable development of rural areas and

$\checkmark$ Optimal utilization of natural resources.

The population in Krushevo according to recent census is in the ranges given in Table 1 (Note 1).

Table 1 . The population in the municipality of Krushevo

\begin{tabular}{cccccccccc}
\hline Municipality Krushevo & Total & Macedonian & Albanian & Vlach & Turkish & Bosnian & Serbian & Roma & Other \\
\hline Total & 9.684 & 6.081 & 2,064 & 1,020 & 315 & 137 & 38 & 0 & 29 \\
& $100,00 \%$ & $62,79 \%$ & $21,31 \%$ & $10,53 \%$ & $3,25 \%$ & $1,41 \%$ & $0,39 \%$ & $0,00 \%$ & $0,30 \%$ \\
\hline
\end{tabular}

The table shows the percentage of representation of minorities in the municipality of Krushevo that should be intermediary in the locomotion of the rural development of this municipality. In this municipality the Macedonian national community dominates. But despite them, significant part are the Albanian community and the Vlach community. Besides the three national communities that dominate in this municipality there are also Turks, Bosnians and Serbs.

\subsection{SWOT Analysis of the Rural Development of the Municipality of Krushevo}

As a basis for setting priorities for rural development in future and define the vision of the municipality of Krushevo it was necessary to perform strategic analysis and diagnosis of the condition. This was done on the basis of the data provided from the Profile of the municipality, taking into account its internal characteristics with the impacts arising from environment. The analysis of strengths, weaknesses, opportunities and threats (SWOT analysis) as a fundamental tool in the process of strategic analysis of the current economic situation, gave opportunity for defining the bases of the key impacts of the internal situation and the external environment on the current and future (desired) state of local community.

The bases for the SWOT analysis were so-called "hard" and "soft" factors of the economic structure of the municipality which belong to the group of internal factors. In the group of direct, that is hard factors of economic structure the labor market, transport connections, natural resources and the characteristics of the land were analyzed. In the group of intermediate or soft factors the factors pertaining to meet the individual needs of citizens, and the factors relating to meeting the specific needs of the enterprise or business environment and the image of the micro location (municipality) and nationwide were taken into consideration. The results of the SWOT - analysis of the municipality of Krushevo along with additional explanations for their choice in the context of the local economic development are presented in the following paragraphs.

Table 2. SWOT analysis of agriculture

\begin{tabular}{ll}
\hline Strengths & Weaknesses \\
\hline -PPR has a very good ground & -Economic power of individual farmers \\
- Healthy products that bring good yield & -Weak transport infrastructure (containers) \\
- Excellent Climatic conditions (water, sun) & -Old machinery \\
- Strategies for Rural Development & -Apathy towards involvement in activities \\
- Advisory service (SBRA, FFRM) & -Small and fragmented plots \\
- Degree of agricultural culture & -Deficit of people to work \\
-Recognition of products (tobacco, Buchin onions, mountain honey) & -Lack of application of DZHP \\
-Available information & -Lack of pedological cards and reonization \\
-Existing of LAG & -Not enough purchasing enterprises \\
-Tradition for agricultural production & -Problems with the inlet / outlet in rural areas \\
- Degree of farmer's culture & \\
\hline Possibilities & Threats \\
\hline -Involvement of youth in agriculture & - Counterfeit products \\
-Branding ofproducts & -Climate changes \\
-Partially covered by the irrigation system & - Inadequate protection \\
- Means for financing to modernize machinery & - Finance for scientific research activity \\
-New Agricultural structures & -Closing foreign markets \\
-Demand of branded / standardized product & - Unloyal competition for purchase \\
\hline
\end{tabular}




\begin{tabular}{ll}
\hline - Opening new markets & - Cancelation of commodity reserves \\
-Usage of RES, positive legal framework & -Existence of pests and diseases \\
-Returning crops as raw materials & -Closure of institutes production \\
-Implanting at regional and national level & -Unregulated / Poor control of imports, of protection of seeds \\
& and tools \\
& -Existence of pollutants \\
\hline
\end{tabular}

From the SWOT analysis of the agriculture in the municipality of Krushevo we can conclude that Krushevo has a great land, excellent climate for cultivation of some agricultural products. Also great the advisory services of APRZ (Note 2) function perfectly and are regularly on site and instruct farmers in the region. The weaknesses are result of the weak purchasing power of the population and the migration of young people in major cities demanding their existence. From the SWOT analysis we can conclude that the involvement of the young people in the agriculture, the opening of new markets, new employment, modernizing agriculture and increasing funding to the sector by the state can be emphasized as a possible opportunity of the of agriculture in Krusevo.

Table 3. SWOT analysis of the environment

\begin{tabular}{ll}
\hline Strengths & Weaknesses \\
\hline - There are natural resources & - Lack of a system for collection and treatment of waste \\
- Existence of LEAP in 9 municipalities including the & - Lack of regional institutional capacity to deal with environmental \\
municipality of Krushevo & - Lack of knowledge, experience, access to international funds \\
- Existence of PCE in all enterprises & - The existence of illegal landfills \\
- Existence of Regional Waste Management & - Low awareness \\
- Existence of national and regional legal framework to protect & - High level of pollution of air \\
the environment & - Lack of monitoring system for monitoring the state of the \\
& environment \\
\hline Possibilities & Threats \\
\hline - Building the capacities in PCE in all municipalities & - Climate Change \\
- Construction of wastewater treatment plants & - Inertness in law enforcement \\
- Construction of heating REK Bitola & - Reduction of budget funds \\
- Construction of public landfills & - Vast use of pesticides in agriculture \\
- Possibility of utilization of funds from international institutions & \\
- Possibility of utilization of renewable energy sources & \\
- Possibility of creating a system for waste management & \\
- Capacity building for environmental protection & \\
\hline
\end{tabular}

From the SWOT analysis of the environment, we can conclude that the existence of natural resources is the strong side, but on the other side or as a potential disadvantage is the non-utilization of these possibilities by the population. Potential opportunities are the construction of water treatment plants, construction of public landfills, opportunities for using renewable energy and so on. As for the threats to the environment as a major threat are the climate changes, reduced budget, legal regulations, etc.

Table 4. SWOT analysis for transport and communication

\begin{tabular}{ll}
\hline Strengths & Weaknesses \\
\hline - Feasibility study and technical documentation for the use of & - Lack of regional pipelines to supply drinking water \\
heat in REK Bitola & - Analyses of road connection region (doen not exist) \\
- The existence of a ground cadastre (digital 100\%) & - Lack of infrastructure for the development of winter tourism \\
- Human Resources & - Lack of integrated pedestrian and bicycle trails in PPR \\
- Geo-strategic position of the region & - Management and traffic control (insufficient vertical and \\
- Gained experience in application to funds & horizontal signaling) \\
\hline Possibilities & Threats \\
\hline - Change of experiences, information and knowledge & - Lack of analysis of traffic connections in the region \\
- Access to other funds & - Impact of the transport infrastructure on the environment \\
- Reconstruction of railway Bitola-Lerin & - Lack of strategy for the development of road transport \\
- Existence of hydrographic potential (Accumulation Strezevo, & infrastructure of URU \\
Prespa Lake ...) & - Lack of relevant data and documentation for infrastructure \\
\hline
\end{tabular}


- Transport steam pipeline the possibility of utilizing thermal energy and greenhouse heating
- Lack of competence at the regional level for road transport infrastructure

- Insufficient use of EU funds

- Lack of underground terrain

SWOT analysis of Transport and Communications as one of the major strengths that are mentioned in the analysis are geostrategic position of the region and the municipal of Krushevo. Unlike this strong side, the weak side is the lack of infrastructure and poor connections with other regions in the Republic of Macedonia. One of the major opportunities that can contribute to the development of this municipality is the access to European funds that can directly contribute to the development of the road network and infrastructure of the municipality. Apart from these opportunities this municipality has potential threats too, and as one of the main threats is the non-utilization of European funds for rural tourism and municipal development.

\subsection{Analysis of the Priority Areas for Rural Development in the Municipality of Krushevo}

From the conducted SWOT analysis and the results of the survey, the priority areas identified are the following sectors:

1). Tourism;

2). Agriculture;

3). Economy;

4). Culture (archaeological sites, events).

The priority areas are identified sectors on which direct actions to improve the situation will be organized. These priorities enable setting high but achievable strategic objectives through the implementation of which the municipality will go towards achieving its vision. In addition, priority areas represent the aspirations and ambitions of citizens, legal entities and municipal administration and future physiognomy of rural areas of the municipality.

It is important to note that each of these areas has equal priority importance for the community and their sequential structure does not mean that it was made and their prioritization of importance.

\section{Priority Area: Tourism}

Strategic Objective 1: Development of rural tourism

Strategic Objective 2: Promotion of tourism offer

Priority Area: Agriculture

Strategic Objective 1: Production of healthy food

Strategic Objective 2: Development of livestock

Priority Area: Economy

Strategic Goal 1: Reduction of unemployment

Strategic Objective 2: Increase of number of small and middle-sized enterprises

Priority area: Culture

Strategic Objective 1: Protection, conservation, restoration and presentation of archaeological and cultural treasures

\section{Vision of the Municipality of Krushevo}

The rural part of the municipality of Krushevo will be ecological, healthy agricultural area, internationally famous cultural destination, desired place for living with high standard of the population achieved through production of healthy food, enterprise and developed forms of tourism and socio cultural life.

\subsection{Analysis of Tourism as a Factor for Rural Development of the Municipality of Krushevo}

Tourism is singled out as one of the priority areas for intervention in the municipality of Krushevo, because of the connection with the development and its role and contribution that it will have in the economic development of the municipality. In establishing tourism as a priority area, the existing conditions and values and connection to other areas and sectors of life were taken into consideration.

To achieve the objective - Development of Sustainable Rural Tourism by providing quality products and services 
implementation of two specific objectives is required:

a. Development of standards for sustainable tourism - this goal would be realized through the establishment of conditions for development of sustainable rural tourism and reinforcement of the local capacities for the needs of rural tourism

b. Identification and promotion of authentic values of the region of the municipality of Krushevo for tourism development, which can be realized by raising awareness on the global changes in tourism and strengthening municipal capacities and the capacities of the local population for the introduction of organized tourism performance.

To achieve the objective - promotion of tourist offer and cultural - historical heritage requires the implementation of two specific objectives:

a. Introduction and promotion of tourist offer and products of rural, alternative and cultural tourism (identification and definition of competitive tourism products and offer rural tourism and promotion of tourist offer of the Municipality of Krusevo), and

b. Preparation and publication of promotional tourist materials for rural municipalities (Prepation promotional printed materials on tourist values of the municipality of Krushevo, Preparation of electronic promotional material on tourist values of the municipality of Krushevo).

\subsection{Analysis of the Agriculture as a Factor for Rural Development of the Municipality of Krushevo}

Agriculture is one of the priority areas for intervention in the municipality of Krushevo, completely and comprehensively as a necessity in the economic development and capacity utilization and the involvement of citizens and the development of the municipality. Agriculture in the function of local economic development provides a complete encircling of the LEADER approach as an official approach for rural development the municipality of Krushevo by strengthening the capacity of the group for rural development for implementation and generating activities for sustainable regional rural development.

To achieve this strategic objective realization of two objectives is required:

a. Establishment of a formal body of the municipality of Krushevo which will work smoothly on sustainable regional rural development which will enable further implementation of the LEADER approach and EU standards,

b. Initiative to create a regional strategy for rural development that will implement the LEADER approach in the region (Development of a regional strategy for GE, which will enable sustainable rural development of the region, preservation of traditional values).

As a subsequent strategic goal in the area of agriculture is the food production that requires the realization of three objectives:

a. Improving conditions for Agricultural Development (Increase the quality and quantity of agricultural product);

b. Development and support of livestock (Increase livestock and preservation of indigenous species and breeds) and,

c. Education of farmers for standardization of products and their classification (Strengthening the capacity of farmers and farmer associations and the use of available financial resources in the field of rural development).

\subsection{Analysis of the Economy as a Factor for Rural Development in the Municipality of Krushevo}

The economic development is a priority and urgent area for intervention in the municipality of Krushevo. Providing perspective, job opportunities and life is essential. The Rural Development Strategy is based on factors related to the field. As a strategic goal of the economy as a priority is the reduction of the unemployment by improving the production conditions of people in the rural regions.

To achieve this strategic goal achievement of the following goals is required:

a. Creating conditions for investment in the economy in the rural part of the municipality (Establishment of a positive climate for investment in the rural economy), and

b. Improving conditions for the development of existing economic entities (Supporting existing economic entities operating in the rural economy).

In realization of the strategic goal - increasing the number of small and middle enterprices with the support and information of the local population and individual farmers and manufacturers, implementation of the following specific objective is required: 
- Strengthening of human resource through education of local people to open new production facilities which will achieve the reduction of unemployment through:

a. Information on all possible uses of human resources and conditions in the community.

b. Use of available financial resources in the field of GE.

c. Developing entrepreneurship among locals.

\subsection{Analysis of Culture as a Factor for Rural Development in the Municipality of Krushevo}

Culture has essential role for the municipality of Krushevo, for the past and the future of this region and it can be the engine of economic and social development of the communities in its integrationand management strengthening activities in this area whose comprehensiveness is included in other strategic objectives.

Therefore, it is priority area that must be built following strategic goal: maximum commitment for protection, conservation and restoration and presentation of archaeological and cultural treasures as the official common value of the municipality of Krushevo.

To achieve this strategic objective implementation of three specific objectives is required:

- Forming a group for rural development that will have a special responsibility in promoting cultural values of the municipality of Krushevo in its activities (mapping and protection of cultural and historical heritage. Availability, stress, awareness and visibility of all archaeological values);

- Complete determination, marking, highlighting and promoting cultural events in the municipality of Krusevo, international cooperation and exchange, amalgamation with other values in harmony with the established guidelines of PDD (Determining and establishing the availability, stress, awareness and visibility of all archaeological values, international cooperation and research) and

- Renovation of existing and construction of new cultural centers, sports fields which will enhance cultural entertaining life (improvement of cultural life in rural areas).

\section{Conclusions}

1) More than a third of the world's population is engaged in agriculture, while three-quarters of the poor inhabit rural areas. Accordingly, the implementation of rural development measures is quite popular in the world and in our country.

2) Establishment of stable rural network in the Republic of Macedonia is very important segment of the rural development, especially because of the equal development of all rural areas.

3) To achieve growth in rural areas it is necessary to develop activities that will create conditions for higher incomes of the population in rural areas in future.

4) Rural development is required in the Republic of Macedonia and the municipality of Krushevo, for approximation to EU standards, the competitiveness of the market and thus improvement of the quality of life of the population living in rural areas.

5) The town of Krushevo as well as all localities that belong to the municipality of Krushevo have a natural potential for rural development. Nature, history, climate, tradition of this municipality offer good conditions for rural development with the correct application of the measures offered by the Republic of Macedonia and the EU in order to improve the quality of life in rural areas.

6) The municipality of Krushevo has good conditions for rural development particularly in agriculture and rural tourism in the town.

7) Republic of Macedonia is a rural country which means that there are ideal conditions for rural development and the European funds should be used maximally

8) Modern rural development policy is geared to defining efficient mechanisms to develop all activities in rural areas, in accordance with the principles of sustainable development and to improve the quality of life of the rural population.

9) Considering that rural development is primarily a structural problem of long-term character, its resolution requires systematic approach.

10) The main objectives and principles of the policy of sustainable rural development in the European Union should be understood as an instrument of modernization, renewal and protection of rural areas. 
11) The responsibility and the importance of local self-government for sustainable rural development in terms of mobilization of local resources and creating an environment to overcome underdevelopment and poverty is great.

12) An active approach from the Municipality of Krushevo to the strategy for local development as strategic operational document is needed, and with this the measures in agriculture, culture, infrastructure and ecology will be successfully realized.

13) Inter - sectorial and inter-regional connectivity and decentralization in the creation and implementation of the strategy and rural development policy have a major impact on the effective activation of local resources, confirming the example on the area of the municipality of Krushevo.

14) The strategic directions that need to move rural development of Krushevo are tourism, economy, agriculture and culture

15) Existing scientific research, including statistical data, do not provide enough inputs for designing a quality support system for rural development in the Republic of Macedonia.

\section{References}

Bryden, J. (2002). Rural Development Indicators and Diversity in the European Union. Conference proceeding: Measuring Rural Diversity. Economic Research Service, Washington, DC, pp. 21-22.

Gogonea, R., Hapenciuc, C., \& Dragan, I. (2009). The link between local and global management in the rural tourism. The Annals of the "Stefan cel Mare" University Suceava, 1(9).

Jafari, J. (2000). Encyclopedia of tourism (p. 514). Routledge.

Jankulovski, N. (2009). Financing the local governments units through loans and pre-accession funds. Master thesis, Institute of Economics - Skopje.

Jankulovski, N. (2015). Analysis of the regions in terms of promoting regional development of Republic of Macedonia. International Journal of Economics and Finance, 7(10). https://doi.org/10.5539/ijef.v7n10p247

Jankulovski, N., Bojkovska, K., \& Mihajlovski, G. (2014). The Financial Conditions and Prospects of the Municipality of Bitola, Republic of Macedonia. International Journal of Economics and Finance, 6(11). http://dx.doi.org/10.5539/ijef.v6n11p197

Jaszczak, A., \& Žukovskis, J. (2010). Tourism business in development of European rural areas. Management Theory and Studies for Rural Business and Infrastructure Development, 20(1).

Mojmir, M., \& Vanco, U. (2005). EU funds for development of the Republic of Macedonia. Governmental Sector for European Integration, Skopje.

National Statistical Office. (n. d.). Retrieved from http://www.stat.gov.mk/pdf/kniga_13.pdf

Richards, G., \& Hall, D. (2000). Tourism and sustainable community development (p. 271). Rout ledge. https://doi.org/10.4324/9780203464915

Šimkova, E. (2007). Strategic approaches to rural tourism and sustainable development of rural area. Agric. Econ. - Czech, 53(6), 263-270.

\section{Notes}

Note 1. http://www.stat.gov.mk/pdf/kniga_13.pdf

Note 2. Agency for stimulating agriculturale.

\section{Copyrights}

Copyright for this article is retained by the author(s), with first publication rights granted to the journal.

This is an open-access article distributed under the terms and conditions of the Creative Commons Attribution license (http://creativecommons.org/licenses/by/4.0/). 